\title{
Outcomes of patients with acute kidney injury with regard to time of initiation and modality of renal replacement therapy - first data from the Silesian Registry of Intensive Care Units
}

\author{
Piotr Czempik ${ }^{1}$, Daniel Cieśla², Piotr Knapik ${ }^{1}$, tukasz Krzych ${ }^{1}$ \\ ${ }^{1}$ Department of Anaesthesiology and Intensive Care, Medical University of Silesia, Silesian Centre for Heart Diseases, Zabrze, \\ Poland \\ ${ }^{2}$ Centre for Research, Training and New Medical Technologies, Silesian Centre for Heart Diseases, Zabrze, Poland
}

Kardiochirurgia i Torakochirurgia Polska 2016; 13 (2): 122-129

\begin{abstract}
Introduction: Acute kidney injury (AKI) remains a serious clinical problem in the intensive care unit (ICU). It constitutes an independent risk factor for mortality, especially when renal replacement therapy (RRT) is required.

Aim: Due to limited evidence pertaining to timing, choice of RRT modality and lack of studies investigating AKI in Polish ICUs, we sought to analyse outcomes of adult AKI-RRT ICU patients in the Silesian Voivodeship.

Material and methods: We analysed data regarding 1,380 patients with AKI who required RRT (AKI-RRT) (9.2\% of all subjects in the registry) hospitalized between October 2011 and December 2014 in Silesian ICUs. The primary outcome was crude ICU mortality. Length of ICU stay (LOS) was considered the secondary outcome.

Results: Of 15,030 patients 1,380 (9.2\%) individuals developed AKI requiring RRT. The overall mortality in the registry was $43.9 \%$, but it was significantly higher (69.1\%) in AKI-RRT patients $(p<0.01)$. Mortality with regard to timing of institution of RRT was $67.1 \%$ in the group with RRT instituted prior to ICU admission (RRT-prior-ICU) and 69.4\% in patients with RRT instituted during ICU hospitalization (RRT-in-ICU) ( $p=0.58$ ).

Conclusions: Multiple patient- and hospitalization-related factors determine mortality in this specific cohort. There are no differences in mortality with regard to RRT being initiated before or during hospitalization in the ICU. Due to multiple confounders, differences in mortality in terms of modality of RRT should be interpreted with caution.
\end{abstract}

Key words: acute kidney injury, renal replacement therapy.

\section{Streszczenie}

Wprowadzenie: Ostre uszkodzenie nerek (acute kidney injury - AKI) jest poważnym problemem klinicznym na oddziale intensywnej terapii. Stanowi niezależny czynnik ryzyka zgonu, zwłaszcza gdy wymaga zastosowania leczenia nerkozastępczego (renal replacement therapy - RRT).

Cel: Niewiele jest prac podejmujących problem czasu włączenia oraz wyboru metody leczenia nerkozastępczego, zwłaszcza u pacjentów hospitalizowanych na polskich oddziałach intensywnej terapii. W związku z tym naszym celem było przeanalizowanie wyników leczenia dorosłych pacjentów z AKI poddawanych leczeniu nerkozastępczemu na oddziałach intensywnej terapii w województwie śląskim.

Materiat i metody: Przeanalizowano dane dotyczące 1380 pacjentów z AKI, którzy wymagali leczenia nerkozastępczego (AKI-RRT) (9,2\% pacjentów w Rejestrze) od października 2011 do grudnia 2014 r. na śląskich oddziałach intensywnej terapii (OIT). Pierwszorzędowym punktem końcowym była śmiertelność na OIT, natomiast drugorzędowym punktem końcowym czas pobytu na OIT.

Wyniki: Spośród 15030 pacjentów u 1380 (9,2\%) wystąpiło AKI wymagające leczenia nerkozastępczego. Ogólna śmiertelność w Rejestrze wynosiła 43,9\% i była znacząco większa $(69,1 \%)$ u pacjentów z AKI poddawanych RRT $(p<0,01)$. Śmiertelność w zależności od czasu włączenia RRT wynosiła $67,1 \%$, gdy leczenie nerkozastępcze włączano przed przyjęciem (RRT-prior-ICU), i 69,4\% w trakcie pobytu (RRT-in-ICU) na OIT $(p=0,58)$. Wnioski: Liczne czynniki dotyczące pacjentów i procesu leczenia w OIT wpływają na śmiertelność w tej specyficznej grupie chorych. Nie stwierdzono różnic w śmiertelności w zależności od czasu włączenia leczenia nerkozastępczego. Z powodu wielu czynników zaburzających różnice w śmiertelności pacjentów w zależności od rodzaju prowadzonej terapii nerkozastępczej powinny być interpretowane z dużą ostrożnością.

Słowa kluczowe: ostre uszkodzenie nerek, terapia nerkozastępcza.

Address for correspondence: Piotr Czempik MD, Department of Anaesthesiology and Intensive Care, Silesian Centre for Heart Diseases, 9 Marii Curie-Sklodowskiej St, 41-800 Zabrze, Poland, phone: +48 505087 875, e-mail: piotr.czempik@wp.pl 


\section{Introduction}

Acute kidney injury (AKI) remains a serious clinical problem in intensive care units (ICU) due to its high frequency, complex pathophysiology and significant impact on the outcomes [1-3]. It constitutes an independent risk factor for mortality, especially when renal replacement therapy (RRT) is required [4].

Multiple factors may influence the prognosis in patients with AKI, including advanced age, worsening of general health status, diminished pre-morbid functional performance, need of hospitalization before ICU admission, late onset of AKI, co-morbidities and their severity [5, 6]. However, Ostermann and Chang [7] found that the outcome of subjects was directly associated with AKI staging, progression of organ failure, nonsurgical admission, and admission following emergency surgery. In the largest multinational, multicentre study of AKI patients in ICU to date, the BEST Kidney trial [8], the investigators found that AKI occurring during hospitalization has exceptionally dismal prognosis.

The outcome of patients with AKI who require RRT (AKIRRT) is even worse because it is usually applied when pharmacological treatment is insufficient or contraindicated [4]. Unfortunately, there are no universal criteria for initiation of RRT in AKI and modality of therapy. The Kidney Disease: Improving Global Outcomes (KDIGO) guidelines only state that RRT is to be initiated emergently when life-threatening disturbances in fluid, electrolyte or acid-base balance exist and recommend considering the broader clinical context (not graded). The KDIGO AKI guidelines also find continuous and intermittent therapies as complementary, although there are clinical situations where continuous therapies are preferable, namely hemodynamic instability, acute brain injury and other causes of increased intracranial pressure [9].

Increasing evidence suggests improved outcome in AKI patients with early institution of RRT [10-12]. However, this question has not been answered by large randomised controlled trials (RCTs) yet. To our knowledge, only one single RCT has been conducted to evaluate the impact of timing of RRT on outcome, and it found no differences in hospital mortality between patients treated early (within 12 hours of oliguria) and patients treated late (i.e. classic indications for RRT) [13].

\section{Aim}

In view of the limited evidence relating to issues of timing of initiation and choice of RRT modality and lack of studies investigating AKI in Polish ICUs, we sought to analyse outcomes of adult AKI-RRT ICU patients in the Silesian Voivodeship.

\section{Material and methods}

This study was carried out as an observational multicentre registry. It comprised adult patients treated in Silesian Voivodeship ICUs in Poland. At the time of data extraction (i.e. December 31, 2014) there were 15,030 records in the registry. All consecutive patients who required RRT during or prior to hospitalization in an ICU, independent of the modality of RRT, were screened ( $n=1566$ ). Subjects with pre-existing end-stage chronic kidney disease were excluded $(n=186)$.

Finally, we analysed data regarding 1,380 patients with AKI who required RRT (AKI-RRT) (9.2\% of all subjects in the registry) hospitalized between October 2011 and December 2014, of whom 146 (10.6\%) individuals had RRT initiated before ICU admission (RRT-prior-ICU) and 1234 (89.4\%) subjects had RRT started during the ICU stay (RRT-in-ICU). The AKI was defined as acute deterioration of kidney function requiring initiation of RRT and corresponds to class 3 of AKI in the AKIN (Acute Kidney Injury Network) classification [9] and class $F$ (Failure) in the RIFLE classification [9]. Initiation of RRT was at the discretion of a treating physician as there was no protocol for initiation of therapy.

Available demographic and clinical data were retrieved. They included 100 variables organized in 24 categories regarding pre-admission period, time of admission and ICU stay. The primary outcome was crude ICU mortality. Length of ICU stay (LOS) was considered the secondary outcome.

The study was approved by the Ethics Committee of the Medical University of Silesia. Due to the non-interventional and anonymous nature of the study, the Ethics Committee waived the requirement for informed consent.

\section{Statistical analysis}

Statistical analysis was performed using licensed MedCalc version 16.1 (MedCalc Software bvba, Ostend, Belgium) statistical software. Continuous variables are presented as median and interquartile range $\left(I Q R\right.$, i.e. $25^{\text {th }}-75^{\text {th }}$ percentile), whereas categorical variables are presented as percentages. All variables were tested for normal distribution using the Shapiro-Wilk test. Between-group differences for continuous variables were assessed using the Kruskal-Wallis test and post-hoc analysis (if applicable). For categorical variables the $\chi^{2}$ test was used. The possible impact of the clinical and demographic parameters on mortality was initially screened by bivariate analyses. Odds ratios (ORs) and $95 \%$ confidence intervals (Cls) were calculated. Variables with a $p$-value $<0.05$ were consecutively subjected to a multivariate analysis. The forward logistic regression method was applied. Logistic ORs with $95 \% \mathrm{Cls}$ were subsequently estimated. The $p$-value $<0.05$ was considered statistically significant.

\section{Results}

Of 15,030 patients 1,380 (9.2\%) individuals developed AKI requiring RRT. RRT was instituted prior to ICU admission in 146 (10.6\%) patients and in 1234 (89.4\%) patients during ICU hospitalization. Median age was 66 (IQR 56-75) years, and there were 495 (35.9\%) females among all AKIRRT patients.

Demographic and clinical data according to timing of initiation and modality of RRT are depicted in detail in Tables I-VI.

The overall mortality in the registry was $43.9 \%$, but it was significantly higher (69.1\%) in AKI-RRT patients $(p<0.01)$. 
Tab. I. Pre-intensive care units (ICU) admission clinical data

\begin{tabular}{|c|c|c|c|c|c|}
\hline Variable & $\begin{array}{c}\text { All } \\
(n=1380)\end{array}$ & $\begin{array}{l}\text { RRT-prior-ICU } \\
\quad(n=146)\end{array}$ & $\begin{array}{l}\text { RRT-in-ICU } \\
(n=1234)\end{array}$ & OR $(95 \% \mathrm{Cl})$ & $P$-value \\
\hline Age [years] & $66(56-75)$ & $66.5(57-74)$ & $66(56-75)$ & $1.02(1.01-1.02)$ & 0.87 \\
\hline Females & $495(35.9)$ & $51(34.9)$ & $444(35.9)$ & $0.96(0.67-1.37)$ & 0.80 \\
\hline Hospitalization prior to ICU [days] & $2(1-8)$ & $7(1-15)$ & $2(1-7)$ & $1.01(0.99-1.03)$ & $<0.001$ \\
\hline Alcohol abuse & $140(10.1)$ & $8(5.5)$ & $132(10.7)$ & $0.48(0.23-1.01)$ & 0.05 \\
\hline Auto-aggressive systemic disease & $38(2.7)$ & $3(2.1)$ & $35(2.8)$ & $0.72(0.22-2.37)$ & 0.59 \\
\hline Malignancies & $62(4.5)$ & $3(2.1)$ & $59(4.8)$ & $0.42(0.13-1.35)$ & 0.14 \\
\hline CAD & $690(50.0)$ & $81(55.5)$ & $609(49.4)$ & $1.28(0.91-1.81)$ & 0.16 \\
\hline DM & $423(30.6)$ & $39(26.7)$ & $384(31.1)$ & $0.81(0.55-1.19)$ & 0.28 \\
\hline Cachexia $\left(\mathrm{BMI}<18.5 \mathrm{~kg} / \mathrm{m}^{2}\right)$ & $51(3.7)$ & $0(0.0)$ & $51(4.1)$ & $0.08(0.00-1.28)$ & 0.07 \\
\hline Arterial hypertension & $726(52.6)$ & $78(53.4)$ & $648(52.5)$ & $1.04(0.74-1.46)$ & 0.83 \\
\hline Previous stroke & $81(5.9)$ & $14(9.6)$ & $67(5.4)$ & $1.85(1.01-3.38)$ & 0.046 \\
\hline Solid organ transplantation & $9(0.6)$ & $1(0.7)$ & $8(0.6)$ & $1.06(0.13-8.51)$ & 0.96 \\
\hline $\mathrm{CHF}$ & $622(45.1)$ & $66(45.2)$ & $556(45.1)$ & $1.01(0.71-1.42)$ & 0.97 \\
\hline CKD & $442(32.0)$ & $58(39.7)$ & $382(31.1)$ & $1.46(1.03-2.08)$ & 0.04 \\
\hline CRF & $130(9.4)$ & $10(6.8)$ & $120(9.7)$ & $0.68(0.35-1.22)$ & 0.26 \\
\hline Chronic neurologic disease & $61(4.4)$ & $8(5.5)$ & $53(4.3)$ & $1.29(0.60-2.77)$ & 0.51 \\
\hline Atherosclerosis & $530(38.4)$ & $65(44.5)$ & $465(37.7)$ & $1.33(0.94-1.88)$ & 0.11 \\
\hline Obesity $\left(\mathrm{BMI}>35 \mathrm{~kg} / \mathrm{m}^{2}\right)$ & $94(6.8)$ & $11(7.5)$ & $83(6.7)$ & $1.13(0.59-2.17)$ & 0.71 \\
\hline \multicolumn{6}{|l|}{ Primary ICU admission diagnosis } \\
\hline Severe sepsis & $249(18.0)$ & 17 (11.6) & $232(18.8)$ & $0.57(0.34-0.96)$ & 0.04 \\
\hline Severe metabolic disorder & $143(10.4)$ & $20(13.7)$ & $123(9.9)$ & $1.43(0.86-2.38)$ & 0.16 \\
\hline Infection & $315(22.8)$ & $38(26.0)$ & $277(22.4)$ & $1.21(0.82-1.80)$ & 0.33 \\
\hline Circulatory insufficiency & $752(54.5)$ & $96(65.7)$ & $656(53.2)$ & $1.69(1.18-2.42)$ & 0.004 \\
\hline MODS & $359(26.0)$ & $36(24.7)$ & $323(26.2)$ & $0.92(0.62-1.37)$ & 0.69 \\
\hline SCA & $278(20.1)$ & $25(17.1)$ & $253(20.5)$ & $0.80(0.51-1.26)$ & 0.34 \\
\hline Acute respiratory failure & $926(67.1)$ & $111(76.0)$ & $815(66.0)$ & $1.63(1.09-2.43)$ & 0.02 \\
\hline Acute neurologic disease & $41(2.9)$ & $5(3.4)$ & $36(2.9)$ & $1.18(0.46-3.06)$ & 0.73 \\
\hline SAP & $60(4.3)$ & $4(2.7)$ & $56(4.5)$ & $0.59(0.21-1.66)$ & 0.32 \\
\hline Post-surgical status & $446(32.3)$ & $69(47.3)$ & $377(30.6)$ & $2.04(1.44-2.88)$ & $<0.001$ \\
\hline TBI & $17(1.2)$ & $0(0.0)$ & $17(1.4)$ & $0.24(0.01-3.97)$ & 0.32 \\
\hline Multiple trauma & $35(2.5)$ & $0(0.0)$ & $35(2.8)$ & $0.11(0.01-1.89)$ & 0.13 \\
\hline Shock & $548(39.7)$ & $56(38.4)$ & $492(39.9)$ & $0.94(0.66-1.33)$ & 0.72 \\
\hline Obtunded consciousness & $512(37.1)$ & $66(45.2)$ & $446(36.1)$ & $1.46(1.03-2.06)$ & 0.03 \\
\hline Acute-on-chronic respiratory failure & $70(5.1)$ & $7(4.8)$ & $63(5.1)$ & $0.94(0.42-2.08)$ & 0.87 \\
\hline Poisoning & $24(1.74)$ & $0(0.0)$ & $24(1.9)$ & $0.17(0.01-2.79)$ & 0.21 \\
\hline \multicolumn{6}{|l|}{ Direct ICU admission diagnosis } \\
\hline Circulatory insufficiency & $1063(77.0)$ & $111(76.0)$ & $952(77.1)$ & $0.94(0.63-1.40)$ & 0.76 \\
\hline Renal failure & $806(58.4)$ & $115(78.8)$ & $691(55.9)$ & $2.92(1.93-4.40)$ & $<0.001$ \\
\hline Respiratory failure & $1217(88.2)$ & $130(89.0)$ & $1087(88.1)$ & $1.09(0.64-1.89)$ & 0.74 \\
\hline Multiple trauma & $40(2.9)$ & $2(1.4)$ & $38(3.1)$ & $0.44(0.10-1.83)$ & 0.26 \\
\hline Metabolic disorders & $579(41.9)$ & $63(43.1)$ & $516(41.8)$ & $1.06(0.75-1.49)$ & 0.76 \\
\hline Obtunded consciousness & $782(56.7)$ & $87(59.6)$ & $695(56.3)$ & $1.14(0.81-1.62)$ & 0.45 \\
\hline
\end{tabular}

CAD - coronary artery disease, DM - diabetes mellitus, BMI - body mass index, CHF - congestive heart failure, CKD - chronic kidney disease, MODS - multiple organ dysfunction syndrome, SCA - sudden cardiac arrest, SAP - severe acute pancreatitis, TBI - traumatic brain injury. 
Tab. II. Clinical data on intensive care units (ICU) admission

\begin{tabular}{lccccc} 
Variable & $\begin{array}{c}\text { All } \\
(n=1380)\end{array}$ & $\begin{array}{c}\text { RRT-prior-ICU } \\
(n=146)\end{array}$ & $\begin{array}{c}\text { RRT-in-ICU } \\
(n=1234)\end{array}$ & OR (95\% CI) & P-value \\
APACHE II & $26(20-32)$ & $26(20-33)$ & $26(20-32)$ & $1.04(1.03-1.06)$ & 0.74 \\
\hline GCS & $7(3-13)$ & $6(3-11)$ & $7(3-13)$ & $0.91(0.89-0.94)$ & 0.14 \\
\hline SAPS III & $64(45-81)$ & $64(43.5-79.5)$ & $64(45-81)$ & $1.01(1.00-1.02)$ & 0.71 \\
\hline 24 h TISS-28 & $39(33-45)$ & $39(34.5-44)$ & $39(33-45)$ & $1.03(1.01-1.04)$ & 0.94 \\
\hline Catecholamines & $833(60.4)$ & $115(78.8)$ & $718(58.2)$ & $2.67(1.76-4.03)$ & $<0.001$ \\
\hline Obtunded & $901(65.3)$ & $103(70.5)$ & $798(64.7)$ & $1.31(0.89-1.90)$ & 0.16 \\
\hline Endocavital stimulation & $21(1.5)$ & $2(1.4)$ & $19(1.5)$ & $0.89(0.20-3.85)$ & 0.87 \\
\hline Mechanical ventilation & $1004(72.7)$ & $122(83.6)$ & $882(71.5)$ & $2.03(1.29-3.19)$ & 0.002 \\
\hline Intubated & $1009(73.1)$ & $122(83.6)$ & $887(71.9)$ & $1.99(1.26-3.13)$ & 0.003 \\
\hline
\end{tabular}

APACHE II - Acute Physiology and Chronic Health Evaluation II, GCS - Glasgow Coma Scale, SAPS - Simplified Acute Physiology Score III, TISS-28 - Therapeutic Intervention Scoring System-28.

Tab. III. Clinical data during intensive care units (ICU) stay

\begin{tabular}{|c|c|c|c|c|c|}
\hline Variable & $\begin{array}{c}\text { All } \\
(n=1380)\end{array}$ & $\begin{array}{l}\text { RRT-prior-ICU } \\
\quad(n=146)\end{array}$ & $\begin{array}{l}\text { RRT-in-ICU } \\
(n=1234)\end{array}$ & OR $(95 \% \mathrm{Cl})$ & P-value \\
\hline Catecholamines & $1290(93.5)$ & $132(90.4)$ & $1158(93.8)$ & $0.62(0.34-1.12)$ & 0.12 \\
\hline Antibiotics & $1282(92.9)$ & $136(93.1)$ & $1146(92.9)$ & $1.04(0.53-2.06)$ & 0.90 \\
\hline ECMO & 27 (1.9) & $6(4.1)$ & $21(1.7)$ & $2.47(0.98-6.24)$ & 0.05 \\
\hline IABP & $95(6.9)$ & $16(10.9)$ & $79(6.4)$ & $1.79(1.02-3.17)$ & 0.04 \\
\hline Intubation & 979 (70.9) & $102(69.9)$ & $877(71.1)$ & $0.94(0.65-1.37)$ & 0.76 \\
\hline NO & $36(2.6)$ & $7(4.8)$ & $29(2.3)$ & $2.09(0.89-4.87)$ & 0.08 \\
\hline Surgery in ICU & $295(21.4)$ & $20(13.7)$ & $275(22.3)$ & $0.55(0.34-0.90)$ & 0.02 \\
\hline Tracheostomy & $324(23.5)$ & $37(25.3)$ & $287(23.3)$ & $1.12(0.75-1.66)$ & 0.57 \\
\hline Invasive ventilation & $1193(86.4)$ & $123(84.2)$ & $1070(86.7)$ & $0.82(0.51-1.32)$ & 0.41 \\
\hline Non-invasive ventilation & $84(6.1)$ & $3(2.1)$ & $81(6.6)$ & $0.29(0.09-0.96)$ & 0.04 \\
\hline
\end{tabular}

ECMO - extracorporeal membrane oxygenation, IABP - intra-aortic balloon pump, NO - nitric oxide.

Mortality with regard to timing of institution of RRT was $67.1 \%$ in the group with RRT instituted prior to ICU admission (RRT-prior-ICU) and 69.4\% in patients with RRT instituted during ICU hospitalization (RRT-in-ICU) ( $p=0.58)$ (Fig. 1). Mortality in internitent hemodialysis (IHD), continuous RRT (CRRT), IHD + CRRT subgroups were 62.2\%, 69.9\%, 74.4\%, respectively ( $p=0.02)$ (Fig. 2).

Median ICU LOS in all AKI-RRT subjects was 9.8 (IQR: 4.0-18.7) days, and it was longer compared to the nonAKI-RRT group (9.8 (IQR: 4.0-19) vs. 5.7 (IQR: 2.1-12) days; $p<0.001$, respectively). Median ICU LOS in the RRT-priorICU group was 9.5 (IQR: 4.2-16.2) and in the RRT-in-ICU group was 9.8 (IQR: 4-18.8). Median ICU LOS in IHD, CRRT and IHD + CRRT groups was 8.6 (IQR: 3.4-17.3), 9.6 (IQR: 3.918.2) and 16.5 (IQR: 8.0-27.3) days, respectively.

After adjustment for timing of initiation and modality of RRT, six variables remained statistically significant predictors of mortality (Table VII). APACHE II score on admission, multiple organ dysfunction syndrome (MODS) as primary admission diagnosis, atherosclerosis in medical history and CRRT as modality of RRT increased mortality. Renal failure as direct admission diagnosis and multiple trauma as primary admission diagnosis reduced the risk of death.

\section{Discussion}

In this comprehensive registry study we sought to investigate the importance of timing of initiation and choice of RRT in AKI patients hospitalised in ICUs in the Silesian Voivodeship.

Based on a large multicentre medical database, we found that the prevalence of AKI requiring RRT in the Silesian Voivodeship was about $10 \%$. This finding is comparable to the results of Nisula (10.2\%) [14] and Uchino (5-6\%) [8].

The AKI subjects receiving RRT constitute a group of patients with particularly dismal prognosis. Mortality of AKIRRT patients reached $62.2-74.4 \%$ depending on the timing of initiation and modality of RRT and was significantly higher than in non-AKI-RRT patients (43.9\% overall mortality in the registry). Hospital mortality of AKI-RRT patients in the present study was higher than in previous studies (Nisula - 25.6\% [14]; Mehta - 37\% [15]; Ostermann - 56.8\% [7]; Uchino - 60\% [8]), which is probably due to different inclusion criteria of RRT administration and study group characteristics. The high hospital mortality of AKI-RRT patients in our study might also be due to high severity of illness as per the APACHE II score of 26 (IQR: 20-32), which corresponds to predicted mortality of 55\% [16]. 
Tab. IV. Pre-intensive care units (ICU) admission clinical data

\begin{tabular}{|c|c|c|c|c|c|}
\hline Variable & $\begin{array}{c}\text { All } \\
(n=1380)\end{array}$ & $\begin{array}{c}\text { IHD } \\
(n=180)\end{array}$ & $\begin{array}{c}\text { CRRT } \\
(n=1118)\end{array}$ & $\begin{array}{c}\text { IHD + CRRT } \\
(n=82)\end{array}$ & $P$-value \\
\hline Age [years] & $66(56-75)$ & $68(57.5-76)$ & $66(56-74)$ & $66.5(52-74)$ & 0.25 \\
\hline Females & $495(35.9)$ & $71(39.4)$ & $399(35.7)$ & $25(30.5)$ & 0.36 \\
\hline Hospitalisation prior to ICU [days] & $2(1-8)$ & $2(0-6)$ & $3(1-8)$ & $1.5(0-6)$ & 0.006 \\
\hline Alcohol abuse & $140(10.1)$ & $16(8.9)$ & $117(10.5)$ & $7(8.5)$ & 0.72 \\
\hline Malignancies & $62(4.5)$ & $9(5.0)$ & $50(4.5)$ & $3(3.7)$ & 0.89 \\
\hline CAD & $690(50.0)$ & $93(51.7)$ & $554(49.6)$ & $43(52.4)$ & 0.78 \\
\hline DM & $423(30.7)$ & $63(35.0)$ & $338(30.2)$ & $22(26.8)$ & 0.32 \\
\hline Cachexia $\left(\mathrm{BMI}<18.5 \mathrm{~kg} / \mathrm{m}^{2}\right)$ & $51(3.7)$ & $4(1.7)$ & $48(4.3)$ & $0(0.0)$ & 0.04 \\
\hline Arterial hypertension & $726(52.6)$ & $92(51.1)$ & $586(52.4)$ & $48(58.5)$ & 0.51 \\
\hline Solid organ transplantation & $9(0.7)$ & $0(0.0)$ & $8(0.7)$ & $1(1.2)$ & 0.44 \\
\hline $\mathrm{CHF}$ & $622(45.1)$ & $77(42.8)$ & $513(45.9)$ & $32(39.0)$ & 0.39 \\
\hline Chronic neurologic disease & $61(4.4)$ & $10(5.6)$ & $48(4.3)$ & $3(3.7)$ & 0.70 \\
\hline Atherosclerosis & $530(38.4)$ & $77(42.8)$ & $412(36.8)$ & $41(50.0)$ & 0.02 \\
\hline Obesity (BMI > 35 kg/m²) & $94(6.8)$ & $14(7.8)$ & $72(6.4)$ & $8(9.8)$ & 0.44 \\
\hline \multicolumn{6}{|l|}{ Primary ICU admission diagnosis } \\
\hline Severe sepsis & $249(18.0)$ & $23(12.8)$ & $208(18.6)$ & $18(21.9)$ & 0.039 \\
\hline Severe metabolic disorder & $143(10.4)$ & $25(13.9)$ & $108(9.7)$ & $10(12.2)$ & 0.190 \\
\hline Infection & $315(22.8)$ & $43(23.9)$ & $246(22.0)$ & $26(31.7)$ & 0.121 \\
\hline Circulatory insufficiency & $752(54.5)$ & $102(56.7)$ & $603(53.9)$ & $47(57.3)$ & 0.688 \\
\hline MODS & $359(26.0)$ & $28(15.6)$ & $308(27.5)$ & $23(28.0)$ & 0.003 \\
\hline SCA & $278(20.1)$ & $47(26.1)$ & $213(19.1)$ & $18(21.9)$ & 0.083 \\
\hline Acute respiratory failure & $926(67.1)$ & $136(75.6)$ & $725(64.8)$ & $65(79.3)$ & 0.001 \\
\hline Acute neurologic disease & $41(3.0)$ & $8(4.4)$ & $30(2.7)$ & $3(3.7)$ & 0.404 \\
\hline SAP & $60(4.3)$ & $4(2.2)$ & $48(4.3)$ & $8(9.8)$ & 0.02 \\
\hline Post-surgical status & $446(32.3)$ & $40(22.2)$ & $377(33.7)$ & $29(35.4)$ & 0.008 \\
\hline TBI & $17(1.2)$ & $2(1.1)$ & $14(1.2)$ & $1(1.2)$ & 0.99 \\
\hline Multiple trauma & $35(2.5)$ & $0(0.0)$ & $33(2.9)$ & $2(2.4)$ & 0.06 \\
\hline Shock & $548(39.7)$ & $69(38.3)$ & $442(39.5)$ & $37(45.1)$ & 0.56 \\
\hline Obtunded consciousness & $512(37.1)$ & $76(42.2)$ & $402(35.9)$ & $34(41.5)$ & 0.19 \\
\hline Acute-on-chronic respiratory failure & $70(5.1)$ & $9(5.0)$ & $58(5.2)$ & $3(3.7)$ & 0.83 \\
\hline Poisoning & $24(1.7)$ & $5(2.8)$ & $16(1.4)$ & $3(3.7)$ & 0.17 \\
\hline \multicolumn{6}{|l|}{ Direct ICU admission diagnosis } \\
\hline Circulatory insufficiency & $1063(77.0)$ & $126(70.0)$ & $879(78.6)$ & $58(70.7)$ & 0.0145 \\
\hline Renal failure & $806(58.4)$ & $108(60.0)$ & $650(58.1)$ & $48(58.5)$ & 0.89 \\
\hline Respiratory failure & $1217(88.2)$ & $166(92.2)$ & $977(87.4)$ & $74(90.2)$ & 0.147 \\
\hline Multiple trauma & $40(2.9)$ & $1(0.6)$ & $37(3.3)$ & $2(2.4)$ & 0.119 \\
\hline Metabolic disorders & $579(42.0)$ & $66(36.7)$ & $470(42.0)$ & $43(52.4)$ & 0.0215 \\
\hline Obtunded consciousness & $782(56.7)$ & $103(57.2)$ & $630(56.3)$ & 49 (59.8) & 0.846 \\
\hline
\end{tabular}

CAD - coronary artery disease, DM - diabetes mellitus, BMI - body mass index, CHF - congestive heart failure, MODS - multiple organ dysfunction syndrome, SCA - sudden cardiac arrest, SAP - severe acute pancreatitis, TBI - traumatic brain injury.

There are different definitions regarding timing of institution of RRT. In our study we considered RRT initiated prior to ICU admission as early and that instituted during hospitalization in the ICU as late RRT. The AKI constitutes an indication for ICU admission, so RRT initiated before admission to the ICU might in fact be considered as early. However, there are no universal and widely accepted definitions for early and late initiation of RRT. In the only RCT in this field, that of Bouman et al. [13], the early RRT group initiated therapy within 12 hours of oliguria defined according to the authors as urine output (UO) $<30 \mathrm{ml} /$ hours for 6 hours not responding to diuretics or hemodynamic optimization or creatinine clearance $(\mathrm{CrCl})<20 \mathrm{ml} / \mathrm{min}$. The same authors defined late RRT when classic indications for RRT were met. 
Tab. V. Clinical data on intensive care units (ICU) admission

\begin{tabular}{lccccc} 
Variable & $\begin{array}{c}\text { All } \\
(n=1380)\end{array}$ & $\begin{array}{c}\text { IHD } \\
(n=180)\end{array}$ & $\begin{array}{c}\text { CRRT } \\
(n=1118)\end{array}$ & $\begin{array}{c}\text { IHD + CRRT } \\
(n=82)\end{array}$ & $\begin{array}{c}\text { P-value } \\
\text { APACHE II }\end{array}$ \\
\hline GCS & $26(20-32)$ & $28(22.5-33.5)$ & $25(19-32)$ & $27(21-32)$ & 0.09 \\
\hline SAPS III & $7(3-13)$ & $6(3-12)$ & $7(3-13)$ & $8(3-14)$ & 0.65 \\
\hline 24 h TISS-28 & $64(45-81)$ & $75(57.5-88)$ & $61.5(41-79)$ & $68.5(57-77)$ & $<0.001$ \\
\hline Catecholamines & $39(33-45)$ & $37(32-41.25)$ & $39(33-45)$ & $36.5(32-41)$ & $<0.001$ \\
\hline Obtunded & $833(60.4)$ & $119(66.1)$ & $667(59.7)$ & $47(57.3)$ & 0.22 \\
\hline Endocavital stimulation & $901(65.3)$ & $126(70.0)$ & $717(64.1)$ & $58(70.7)$ & 0.17 \\
\hline Mechanical ventilation & $21(1.5)$ & $1(0.6)$ & $17(1.5)$ & $3(3.7)$ & 0.16 \\
\hline Intubated & $1004(72.8)$ & $146(81.1)$ & $799(71.5)$ & $59(71.9)$ & 0.03 \\
\hline APACHE - Acul $)$ & $144(80.0)$ & $804(71.9)$ & $61(74.4)$ & 0.07 \\
\hline
\end{tabular}

APACHE II - Acute Physiology and Chronic Health Evaluation II, GCS - Glasgow Coma Scale, SAPS - Simplified Acute Physiology Score III, TISS-28 - Therapeutic Intervention Scoring System-28.

Tab. VI. Clinical data during intensive care units (ICU) stay

\begin{tabular}{|c|c|c|c|c|c|}
\hline Variable & $\begin{array}{c}\text { All } \\
(n=1380)\end{array}$ & $\begin{array}{c}\text { IHD } \\
(n=180)\end{array}$ & $\begin{array}{c}\text { CRRT } \\
(n=1118)\end{array}$ & $\begin{array}{c}\text { IHD + CRRT } \\
(n=82)\end{array}$ & $P$-value \\
\hline Catecholamines & $1290(93.5)$ & $163(90.6)$ & $1049(93.8)$ & $78(95.1)$ & 0.21 \\
\hline Antibiotics & $1282(92.9)$ & $167(92.8)$ & $1040(93.0)$ & 75 (91.5) & 0.87 \\
\hline ECMO & $27(2.0)$ & $0(0.0)$ & $26(2.3)$ & $1(1.2)$ & 0.09 \\
\hline IABP & $95(6.9)$ & $4(2.2)$ & $89(7.9)$ & $2(2.4)$ & 0.0049 \\
\hline Intubation & 979 (70.9) & $131(72.8)$ & 781 (69.9) & $67(81.7)$ & 0.06 \\
\hline NO & $36(2.6)$ & $0(0.0)$ & $35(3.1)$ & $1(1.2)$ & 0.04 \\
\hline Surgery in ICU & $295(21.4)$ & $24(13.3)$ & $250(22.4)$ & $21(25.6)$ & 0.01 \\
\hline Tracheostomy & $324(23.5)$ & $33(18.3)$ & $259(23.2)$ & $32(39.0)$ & 0.001 \\
\hline Invasive ventilation & $1193(86.4)$ & $155(86.1)$ & $963(86.1)$ & $75(91.5)$ & 0.39 \\
\hline
\end{tabular}

ECMO - extracorporeal membrane oxygenation, IABP - intra-aortic balloon pump, NO - nitric oxide.

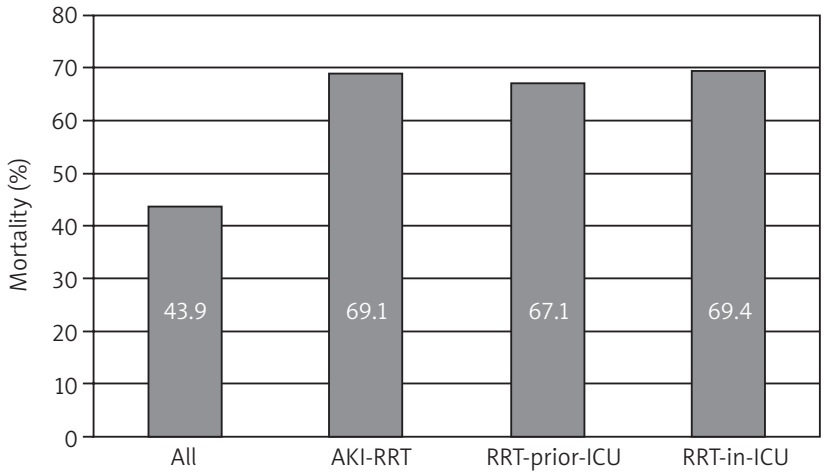

Fig. 1. Mortality of patients according to time of RRT institution. $P<0.05$ : all vs. AKI-RRT; all vs. RRT-prior-ICU; all vs. RRT-in-ICU

The investigators did not find any differences in ICU or hospital mortality between early and late RRT groups. These results reflects our findings, although definitions for timing of RRT were different. In some prospective observational studies it was found that initiation of RRT at lower blood urea nitrogen (BUN) concentrations or closer to ICU admission is beneficial for survival. In the PICARD study [7] initiation of RRT at BUN > $76 \mathrm{mg} / \mathrm{dl}$ was associated with higher

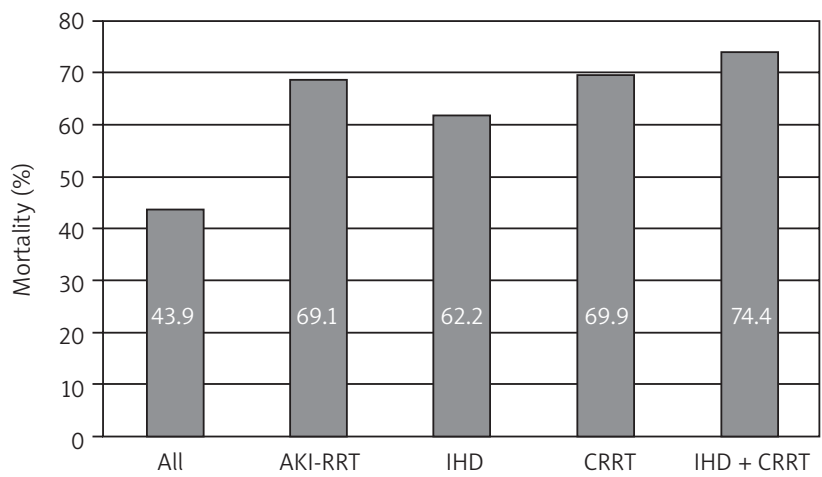

Fig. 2. Mortality of patients according to RRT modality. $P<0.05$ : all vs. AKI-RRT; all vs. IHD; all vs. CRRT; all vs. IHD + CRRT

mortality $(\mathrm{OR}=1.85 ; 95 \% \mathrm{Cl}: 1.16-2.96)$. In the study by Bagshaw et al. [10], early RRT was initiated at BUN $<67.8 \mathrm{mg} / \mathrm{dl}$ ( $24.2 \mathrm{mmol} / \mathrm{l})$, and timing of RRT was also categorized temporally from ICU admission into early ( $<2$ days), delayed (2-5 days) and late ( $>5$ days). The authors found no difference in mortality with regards to BUN concentration, but late initiation of RRT as per temporal categories was associated with higher crude mortality, longer RRT, longer 
Tab. VII. Multiple regression analysis

\begin{tabular}{lcc} 
Independent variable & $\begin{array}{c}\text { Coefficient } \pm \\
\text { standard error }\end{array}$ & $P$-value \\
Modality of RRT & $0.07 \pm 0.04$ & 0.045 \\
\hline $\begin{array}{l}\text { Direct admission diagnosis - } \\
\text { renal failure }\end{array}$ & $-0.11 \pm 0.04$ & 0.005 \\
\hline Atherosclerosis & $0.09 \pm 0.04$ & 0.012 \\
\hline Primary admission diagnosis - MODS & $0.13 \pm 0.05$ & 0.006 \\
\hline $\begin{array}{l}\text { Primary admission diagnosis - } \\
\text { multiple trauma }\end{array}$ & $-0.24 \pm 0.10$ & 0.023 \\
\hline APACHE II & $0.01 \pm 0.002$ & $<0.001$ \\
\hline
\end{tabular}

ICU LOS and greater dialysis dependence. Late initiation of RRT as per RIFLE criteria was identified as an independent predictor of mortality [17]. Disagreement of these results with the findings from our study might be due to different pathophysiology of AKI present before and on admission to the ICU and occurring late during hospitalization in the ICU.

Regarding RRT modality, the findings from our study favour IHD as opposed to CRRT (crude hospital mortality 62.2 vs. $69.9 \%$ ). Although there are some differences between IHD and CRRT, hard end-points of most studies do not favour any particular RRT modality. A meta-analysis comprising 1550 patients found no differences between CRRT and IHD as regards hospital mortality, ICU mortality, LOS and renal recovery [18]. However, some observational studies suggest that CRRT may be beneficial in restoring renal function in survivors [19]. One should bear in mind that IHD and CRRT groups differed significantly in terms of Therapeutic Intervention Scoring System-28 (TISS-28), intra-aortic balloon pump (IABP), nitric oxide (NO) use. The CRRT was also a treatment of choice in more haemodynamically compromised patients (Tab. II, III). Therefore, the differences in mortality should be interpreted with caution.

Apart from RRT modality, we found other determinants of compromised outcome in AKI-RRT patients (Tab. V). The AKI-RRT patients with higher severity of illness are more likely to die, which in our study is exemplified by increased risk for mortality in patients with MODS as the primary admission diagnosis and in patients with a higher APACHE II score. We found multiple trauma as the primary admission diagnosis to reduce mortality in this cohort of patients, which corresponds to the results of other authors [20].

Fluid management also contributes to the course and outcome of AKI. Colloids should be avoided at all costs, especially in shock patients [21]. There is also a U-shaped association between fluid balance and risk of AKI with corresponding mortality $[22,23]$. Fluid overload is a confirmed, independent factor increasing incidence and severity of AKI [24]. Moreover, higher cumulative fluid balance is associated with short-term mortality following AKI [24, 25]. Therefore, the early therapeutic intervention and diagnostic approach plays a key role in improving prognosis in AKI patients. Even minor increases in creatinine concentration are associated with adverse outcomes [26]. Novel biomarkers are suggested to expedite diagnosis of AKI [27].
Our study has several limitations. First, we lacked some important clinical data, including creatinine concentration, fluid balance, timing of RRT, which might have influenced the outcome in the AKI-RRT population. However, entering all these additional parameters into a large database would take enormous effort on the part of attending physicians and potentially discourage them from reporting. One has to bear in mind that reporting was entirely voluntary and the registry was not set up to specifically research AKI. Second, there were no accurate definitions for some conditions given, e.g. a broad definition of shock without specifying what type should be considered: cardiogenic, haemorrhagic, anaphylactic, etc. Finally, the observational nature of our project is an obvious limitation of all registries, as it may lead to a systematic error. However, the large number of patients analysed may compensate for this drawback. Being aware of all shortcomings, the above-presented clinical evidence should attract the attention of an international audience, as it is the first such profound insight into the problem of AKI-RRT in Polish ICUs.

\section{Conclusions}

Prognosis of patients with acute kidney injury and receiving renal replacement therapy in Silesian ICUs is unfavourable. Multiple patient- and hospitalization-related factors determine mortality in this specific cohort. There are no differences in mortality with regard to RRT being initiated before or during hospitalization in the ICU. Due to multiple confounders, differences in outcome in terms of modality of RRT should be interpreted with caution.

\section{Disclosure}

Authors report no conflict of interest.

\section{References}

1. Hoste EA, Bagshaw SM, Bellomo R, Cely CM, Colman R, Cruz DN, Edipidis K, Forni LG, Gomersall CD, Govil D, Honoré PM, Joannes-Boyau O, Joannidis $\mathrm{M}$, Korhonen AM, Lavrentieva A, Mehta RL, Palevsky P, Roessler E, Ronco C, Uchino S, Vazquez JA, Vidal Andrade E, Webb S, Kellum JA. Epidemiology of acute kidney injury in critically ill patients: the multinational AKI-EPI study. Intensive Care Med 2015; 41: 1411-1423.

2. Case J, Khan S, Khalid R, Khan A. Epidemiology of acute kidney injury in the intensive care unit. Crit Care Res Pract 2013; 2013: 479730.

3. Oeyen S, De Corte W, Benoit D, Annemans L, Dhondt A, Vanholder R, Decruyenaere J, Hoste E. Long-term quality of life in critically ill patients with acute kidney injury treated with renal replacement therapy: a matched cohort study. Crit Care 2015; 19: 289.

4. Iwagami M, Yasunaga H, Noiri E, Horiguchi H, Fushimi K, Matsubara T, Yahagi N, Nangaku M, Doi K. Current state of continuous renal replacement therapy for acute kidney injury in Japanese intensive care units in 2011: analysis of a national administrative database. Nephrol Dial Transplant 2015; 30: 988-995.

5. Brivet FG, Kleinknecht DJ, Loirat P, Landais PJ. Acute renal failure in intensive care units: causes, outcome, and prognostic factors of hospital mortality; a prospective, multicenter study. French Study Group on Acute Renal Failure. Crit Care Med 1996; 24: 192-198.

6. Poukkanen M, Vaara ST, Reinikainen M, Selander T, Nisula S, Karlsson S, Parviainen I, Koskenkari J, Pettilä V; FINNAKI Study Group. Predicting one-year mortality of critically ill patients with early acute kidney injury: data from the prospective multicenter FINNAKI study. Crit Care 2015; 19: 125.

7. Ostermann M, Chang RW. Acute kidney injury in the intensive care unit according to RIFLE. Crit Care Med 2007; 35: 1837-1843. 
8. Uchino S, Kellum JA, Bellomo R, Doig GS, Morimatsu H, Morgera S, Schetz M, Tan I, Bouman C, Macedo E, Gibney N, Tolwani A, Ronco C, Beginning and Ending Supportive Therapy for the Kidney (BEST Kidney) Investigators. Acute renal failure in critically ill patients: a multinational, multicenter study. JAMA 2005; 294: 813-818.

9. Kidney Disease: Improving Global Outcomes (KDIGO) Acute Kidney Injury Work Group. KDIGO Clinical Practice Guideline for Acute Kidney Injury. Kidney Int Suppl 2012; 2: 1-138.

10. Bagshaw SM, Uchino S, Bellomo R, Morimatsu H, Morgera S, Schetz M, Tan I, Bouman C, Macedo E, Gibney N, Tolwani A, Oudemans-van Straaten HM, Ronco C, Kellum JA, Beginning and Ending Supportive Therapy for the Kidney (BEST Kidney) Investigators. Timing of renal replacement therapy and clinical outcomes in critically ill patients with severe acute kidney injury. J Crit Care 2009; 24: 129-140.

11. Leite TT, Macedo E, Pereira SM, Bandeira SR, Pontes PH, Garcia AS, Militão FR, Sobrinho IM, Assunção LM, Libório AB. Timing of renal replacement therapy initiation by AKIN classification system. Crit Care 2013; 17: R62.

12. Thakar CV, Rousseau J, Leonard AC. Timing of dialysis initiation in AKI in ICU: international survey. Crit Care 2012; 16: R237.

13. Bouman CS, Oudemans-Van Straaten HM, Tijssen JG, Zandstra DF, Kesecioglu J. Effects of early high-volume continuous venovenous hemofiltration on survival and recovery of renal function in intensive care patients with acute renal failure: a prospective, randomized trial. Crit Care Med 2002; 30: 2205-2211.

14. Nisula S, Kaukonen KM, Vaara ST, Korhonen AM, Poukkanen M, Karlsson S, Haapio M, Inkinen O, Parviainen I, Suojaranta-Ylinen R, Laurila JJ, Tenhunen J, Reinikainen M, Ala-Kokko T, Ruokonen E, Kuitunen A, Pettilä V, FINNAKI Study Group. Incidence, risk factors and 90-day mortality of patients with acute kidney injury in Finnish intensive care units: the FINNAKI study. Crit Care Med 2013; 39: 420-428.

15. Mehta RL, Pascual MT, Soroko S, Savage BR, Himmelfarb J, Ikizler TA, Paganini EP, Chertow GM; Program to Improve Care in Acute Renal Disease. Spectrum of acute renal failure in the intensive care unit: the PICARD experience. Kidney Int 2004; 66: 1613-1621.

16. Knaus WA, Draper EA, Wagner DP, Zimmerman JE. APACHE II: a severity of disease classification system. Crit Care Med 1985; 13: 818-829.

17. Shiao CC, Wu VC, Li WY, Lin YF, Hu FC, Young GH, Kuo CC, Kao TW, Huang DM, Chen YM, Tsai PR, Lin SL, Chou NK, Lin TH, Yeh YC, Wang CH, Chou A, Ko WJ, Wu KD, National Taiwan University Surgical Intensive Care Unit-
Associated Renal Failure Study Group. Late initiation of renal replacement therapy is associated with worse outcomes in acute kidney injury after major abdominal surgery. Crit Care 2009; 13: R171.

18. Rabindranath $\mathrm{K}$, Adams J, Macleod AM, Muirhead N. Intermittent versus continuous renal replacement therapy for acute renal failure in adults. Cochrane Database Syst Rev 2007; 3: CD003773.

19. Bell M, Granath F, Schon S, Ekbom A, Martling CR. Continuous renal replacement therapy is associated with less chronic renal failure than intermittent haemodialysis after acute renal failure. Intensive Care Med 2007; 33: 773-780.

20. Beitland S, Moen H, Os I. Acute kidney injury with renal replacement therapy in trauma patients. Acta Anaesthesiol Scand 2010; 54: 833-840.

21. Rochwerg B, Alhazzani W, Gibson A, Ribic CM, Sindi A, Heels-Ansdell D, Thabane L, Fox-Robichaud A, Mbuagbaw L, Szczeklik W, Alshamsi F, Altayyar S, Ip W, Li G, Wang M, Włudarczyk A, Zhou Q, Annane D, Cook DJ, Jaeschke R, Guyatt GH; FISSH Group (Fluids in Sepsis and Septic Shock). Fluid type and the use of renal replacement therapy in sepsis: a systematic review and network meta-analysis. Intensive Care Med 2015; 41: 1561-1571.

22. Vaara ST, Korhonen AM, Kaukonen KM, Nisula S, Inkinen O, Hoppu S, Lau rila JJ, Mildh L, Reinikainen M, Lund V, Parviainen I, Pettilä V; FINNAKI Study Group. Fluid overload is associated with an increased risk for 90-day mortality in critically ill patients with renal replacement therapy: data from the prospective FINNAKI study. Crit Care 2012; 16: R197.

23. Bellamy MC. Wet, dry or something else? Br J Anaesth 2006; 97: 755-757.

24. Xiong J, Tang X, Hu Z, Nie L, Wang Y, Zhao J. The RIFLE versus AKIN classification for incidence and mortality of acute kidney injury in critical ill patients: a meta-analysis. Sci Rep 2015; 5: 17917.

25. Teixeira C, Garzotto F, Piccinni P, Brienza N, lannuzzi M, Gramaticopolo S, Forfori F, Pelaia P, Rocco M, Ronco C, Anello CB, Bove T, Carlini M, Michetti V, Cruz DN; NEFROlogia e Cura INTensiva (NEFROINT) investigators. Fluid balance and urine volume are independent predictors of mortality in acute kidney injury. Crit Care 2013; 17: R14.

26. Kork F, Balzer F, Spies CD, Wernecke KD, Ginde AA, Jankowski J, Eltzschig HK. Minor postoperative increases of creatinine are associated with higher mortality and longer hospital length of stay in surgical patients. Anesthesiology 2015; 123: 1301-1311.

27. Gavrić A, Kališnik JM. Novel biomarkers for early diagnosis of acute kidney injury after cardiac surgery in adults. Kardiochir Torakochirurgia Pol 2016; 13: 31-38. 